\title{
For dietary advice in end-stage liver cirrhosis resting metabolic rate should be measured, not estimated
}

\author{
Daphne Bot ${ }^{1}$, Anneke Droop', Maarten E. Tushuizen², Bart van Hoek ${ }^{2}$ \\ 'Department of Dietetics, Leiden University Medical Center, Leiden 2300 RC, The Netherlands. \\ ${ }^{2}$ Department of Gastroenterology and Hepatology, and Transplantation Center, Leiden University Medical Center, Leiden 2300 \\ RC, The Netherlands.
}

Correspondence to: Daphne Bot, Department of Dietetics, Leiden University Medical Center, Leiden 2300 RC, The Netherlands. E-mail: d.bot@lumc.nl

How to cite this article: Bot D, Droop A, Tushuizen ME, van Hoek B. For dietary advice in end-stage liver cirrhosis resting metabolic rate should be measured, not estimated. Hepatoma Res2020;6:45. http://dx.doi.org/10.20517/2394-5079.2020.62

Received: 24 Jun 2020 Accepted: 28 Jun 2020 Published: 10 Jul 2020

Academic Editor: Guang-Wen Cao Copy Editor: Cai-Hong Wang Production Editor: Tian Zhang

Dear Editor,

We read with great interest the article "Frailty and Liver resection: where do we stand?" by Sioutas et al. ${ }^{[1]}$ in Hepatoma Research. In this review, the authors summarized the available frailty tools and their impact on postoperative outcomes in patients undergoing liver resection, in particular elderly patients ( $>60$ years). In addition to this review, we would like to provide extra information regarding this topic based on own research.

One of the variables included in most frailty assessments is nutritional status, e.g., loss of body weight or muscle mass. It is well known that malnutrition has a negative impact on clinical outcome of patients with end-stage liver cirrhosis. Insufficient nutrient intake, impaired digestion or absorption of nutrients, and disturbances in macronutrient metabolism contribute to malnutrition in these patients. The total energy expenditure consists of resting metabolic rate (RMR) and expenditure for physical activity. The Harris and Benedict $(\mathrm{HB})$ equation is widely used in clinical care for estimating $\mathrm{RMR}^{[2]}$. However, estimating RMR with HB may be unreliable in patients with cirrhosis. These patients can be hyper- or hypometabolic with many individual differences in energy expenditure, especially based on disease severity and body composition. Measuring RMR in a respiratory chamber is reliable but cumbersome ${ }^{[3]}$. Cheaper and less complicated devices to perform indirect calorimetry measurements have become available. We compared estimated RMR derived with the HB equation with measured RMR using desktop indirect calorimetry in patients with endstage liver cirrhosis. 
After obtaining informed consent from 29 consecutive patients with cirrhosis and preparing for liver transplantation, RMR was measured with desktop indirect calorimetry (Fitmate $\odot$, Cosmed) and compared to the results estimated by the Harris and Benedict equation. Twenty-nine patients ( $79.3 \%$ male) with liver cirrhosis had a mean $( \pm 1.96 \mathrm{SD})$ estimated RMR with HB equation of $1771( \pm 253)$ kilocalories, while the mean measured RMR with Fitmate was $1,630( \pm 322)$ kilocalories $(P<0.05)$. The mean $( \pm 1.96 \mathrm{SD})$ difference in RMR was $140( \pm 240)$ kilocalories, with a minimum of -424 and a maximum of 510 kilocalories difference. The Pearson correlation between measured and estimated RMR was $\mathrm{R}=0.677(P<0.05)$, which is a significant but not strong correlation [Supplementary Figure 1]. Large clinically relevant differences were detected between measured and estimated RMR in patients with liver cirrhosis during screening for liver transplantation. The most likely explanation for the discrepancy is the altered body composition and the frequent presence of ascites in these patients. A limitation of the device used was that it measures $\mathrm{VO}_{2}$ but calculates $\mathrm{VCO}_{2}$. Indirect calorimetry devices that measure both $\mathrm{VCO}_{2}$ and $\mathrm{VO}_{2}$ are even more accurate ${ }^{[2]}$.

In conclusion, for reliable dietary advice in patients with end-stage liver cirrhosis, RMR should be measured with one of these newer easy-to-use devices, and should no longer be estimated with HB and other equations. This can have potential beneficial effects on nutritional status and therefore frailty in patients with liver diseases.

\section{DECLARATIONS}

\section{Acknowledgments}

We thank Mrs. Suzanne van Keeken, MSc and Anneke S. Donker for performing the measurements.

\section{Authors' contributions}

Made substantial contributions to conception and design of the study and performed data analysis and interpretation: Bot D, Droop A, Tushuizen ME, van Hoek B

\section{Availability of data and materials}

Not applicable.

\section{Financial support and sponsorship}

None.

\section{Conflicts of interest}

All authors declared that there are no conflicts of interest.

\section{Ethical approval and consent to participate}

Not applicable.

\section{Consent for publication}

Not applicable.

\section{Copyright}

(c) The Author(s) 2020.

\section{REFERENCES}

1. Sioutas GS, Ziogas IA, Tsoulfas G. Frailty and liver resection: where do we stand? Hepatoma Res 2020;6:4.

2. Roza AM, Shizgal HM. The harris benedict equation reevaluated: resting energy requirements and the body cell mass. Am J Clin Nutr 1984;40:168-82.

3. Verboeket-van der Venne WPHG, Westerterp KR, van Hoek B, Swart GR. Energy expenditure and substrate metabolism in patients with cirrhosis of the liver: effects of the pattern of food intake. Gut 1995;36:110-6.

4. Purcell SA, Elliott SA, Ryan AM, Sawyer MB, Prado CM. Accuracy of a portable indirect calorimeter for measuring resting energy expenditure in individuals with cancer. JPEN J Parenter Enteral Nutr 2019;43:145-51. 